\title{
Analysis and Improvement of Lindell's UC-Secure Commitment Schemes
}

\author{
Olivier Blazy ${ }^{1}$, Céline Chevalier ${ }^{3}$, David Pointcheval ${ }^{2}$, and Damien Vergnaud ${ }^{2}$ \\ 1 Ruhr-Universität Bochum, Germany \\ 2 ENS, Paris, France ${ }^{\dagger}$ \\ 3 Université Panthéon-Assas, Paris, France
}

\begin{abstract}
In 2011, Lindell proposed an efficient commitment scheme, with a non-interactive opening algorithm, in the Universal Composability (UC) framework. He recently acknowledged a bug in its security analysis for the adaptive case. We analyze the proof of the original paper and propose a simple patch of the scheme. More interestingly, we then modify it and present a more efficient commitment scheme secure in the UC framework, with the same level of security as Lindell's protocol: adaptive corruptions, with erasures. The security is proven in the standard model (with a Common Reference String) under the classical Decisional Diffie-Hellman assumption. Our proposal is the most efficient UC-secure commitment proposed to date (in terms of computational workload and communication complexity).
\end{abstract}

Keywords. Universal Composability, Commitment, Efficiency

\section{Introduction}

Related Work. The Universal Composability (UC) framework introduced by Canetti [Can01] is a popular security paradigm. It guarantees that a protocol proven secure in this framework remains secure even if it is run concurrently with arbitrary - even insecure - protocols (whereas classical definitions only guarantee its security in the stand-alone setting). The UC framework enables one to split the design of a complex protocol into that of simpler sub-protocols.

Commitment schemes are one of the most important tools in cryptographic protocols. This is a twophase protocol between two parties, a committer and a receiver. In the first commit phase, the committer gives the receiver an in silico analogue of a sealed envelope containing a value $m$. In the second opening phase, the committer reveals $m$ in such a way that the receiver can verify it. As in the sealed envelope analogy, it is required that a committer cannot change the committed value (i.e., he should not be able to open to a value different from the one he committed to), this is called the binding property. It is also required that the receiver cannot learn anything about $m$ before the opening phase, this is simply called the hiding property.

The security definition for commitment schemes in the UC framework was presented by Canetti and Fischlin [CF01]. A UC-secure commitment scheme achieves the binding and hiding properties under any concurrent composition with arbitrary protocols and it was shown, in [CF01], that it cannot be securely realized without additional assumptions. The common reference string (CRS) setting is the most widely used assumption when considering commitment schemes. In this setting, all parties have access to public information ideally drawn from some predefined distribution.

From a theoretical viewpoint, UC-secure commitments are an essential building block to construct more complex UC-secure protocols such as zero-knowledge protocols [DN02] and two-party or multiparty computations [CLOS02]. Moreover, a UC-secure commitment scheme provides equivocability (i.e., an algorithm that knows a secret related to the CRS can generate commitments that can be opened correctly to any value) and extractability (i.e., another algorithm that knows a secret related to the CRS can correctly extract the content of any valid commitment generated by anybody). Therefore, since their introduction, UC-secure commitments have found numerous practical applications in the area of Authenticated Key Exchange, either in Password Authenticated Key Exchange like [GL03, $\mathrm{CHK}^{+} 05$, ACP09], or the recent generalization to Language Authenticated Key Exchange [BBC $\left.{ }^{+} 13\right]$.

$\bar{\dagger}$ ENS, CNRS \& INRIA - UMR 8548 
Several UC-secure commitment schemes in the CRS model have been proposed. Canetti and Fischlin [CF01] and Canetti, Lindell, Ostrovsky, and Sahai [CLOS02] proposed inefficient non-interactive schemes from general primitives. On the other hand, Damgård and Nielsen [DN02], and Camenish and Shoup [CS03] (among others) presented interactive constructions from several number-theoretic assumptions.

Lindell [Lin11a] has recently presented the first very efficient commitment schemes proven in the UC framework. They can be viewed as combinations of Cramer-Shoup encryption schemes and $\Sigma$-protocols. He presented two versions, one proven against static adversaries (static corruptions), while the other can also handle adaptive corruptions. These two schemes have commitment lengths of only 4 and 6 group elements respectively, while their total communication complexity amount to 14 and 19 group elements respectively. Their security relies on the classical Decisional Diffie-Hellman assumption in standard cryptographic groups. Fischlin, Libert and Manulis [FLM11] shortly after adapted the scheme secure against static corruptions by removing the interaction in the $\Sigma$-protocol using non-interactive Groth-Sahai proofs [GS08]. This transformation also makes the scheme secure against adaptive corruptions but at the cost of relying on the Decisional Linear assumption in symmetric bilinear groups. It thus requires the use of computationally expensive pairing computations for the receiver and can only be implemented over groupes twice ${ }^{1}$ as large (rather than the ones that do not admit pairing computations).

Contributions of the paper. Recently, Lindell edited the ePrint version of his paper [Lin11b], to signal a bug in the proof of the protocol design for adaptive corruptions. While there is no known detail on this bug, we detail on this paper a possible inconsistency on the binding property of the scheme. In order to avoid the above concern, we propose a simple patch to Lindell's scheme making it secure against adaptive corruptions.

However, our main contribution is on improving both Lindell's commitment schemes [Lin11a]. As mentioned above, the committer encrypts the value $m$ (encoded as a group element) using the CramerShoup encryption scheme [CS98]. In the opening phase, he simply reveals the value $m$ and uses a $\Sigma$ protocol to give an interactive proof that the message is indeed the one encrypted in the ciphertext. In Lindell's schemes, the challenge in the $\Sigma$ protocol is sent to the committer using a "dual encryption scheme". Our improvement consists in noting that the receiver can in fact send this challenge directly without having to send it encrypted before. With additional modifications of the schemes, we can present two new protocols secure under the DDH assumption in the UC framework, against static and adaptive corruptions. Both schemes requires a smaller bandwidth and less interactions than the original schemes:

- Static corruptions: the scheme requires the communication of 9 group elements and 3 scalars where Lindell's original proposal requires 10 group elements and 4 scalars. The commit phase is non-interactive and the opening phase needs 3 rounds (instead of 5 in Lindell's scheme).

- Active corruptions: the scheme requires the communication of 10 group elements and 4 scalars where Lindell's original proposal requires 12 group elements and 6 scalars. The commitment phase needs 3 rounds (instead of 5 in Lindell's scheme) and the opening phase is non-interactive.

Implemented on suitable elliptic curves over 256-bit finite fields, our schemes provide a 128-bit security level with a communication complexity reduced to only 3072 and 3584 bits respectively (see Table 1 for a detailed comparison). The computational workload of the new schemes has also slightly decreased compared to Lindell's original proposal and significantly better than Fischlin et al.'s scheme from [FLM11] since the new schemes do not require any expensive pairing computation and can be implemented in much smaller groups.

Outline of the Paper. We start by reviewing the standard definitions, in Section 2. We then present the original Lindell's commitment schemes in Section 3, followed by an explanation of a possible inconsistency and a simple correction in Section 4 .

\footnotetext{
${ }^{1}$ It may be possible to adapt the scheme from [FLM11] to asymmetric bilinear groups using the instantiation of Groth-Sahai proofs based on the Strong eXternal Diffie-Hellman assumption but our scheme will nevertheless remain more efficient.

2 These numbers can be reduced using batching techniques $\left[\mathrm{BFI}^{+} 10\right]$ but at the cost of additional exponentiations.
} 
Section 5 focuses on improving the original protocols. We will show how to reduce both the number of rounds and the number of elements exchanged, in both schemes. We then provide complete security proofs under the same computational assumption as for the original schemes, namely the DDH assumption.

\section{Definitions}

\subsection{Commitments}

A commitment scheme $\mathcal{C}$ is defined by 3 algorithms:

- $\operatorname{Setup}\left(1^{\mathfrak{K}}\right)$, where $\mathfrak{K}$ is the security parameter, generates the global parameters param of the scheme, implicitly given as input to the other algorithms;

- Commit $(m ; r)$ produces a commitment $c$ on the input message $m \in \mathcal{M}$, using the random coins $r \stackrel{\$}{\leftarrow} \mathcal{R}$, and also outputs the opening information $w$;

- Decommit $(c, m ; w)$ decommits the commitment $c$ using the opening information $w$; it outputs the message $m$, or $\perp$ if the opening check fails.

Such a scheme should be both binding, which means that the decommit phase can successfully open to one value only, and hiding, which means that the commit phase does not reveal any information about $m$.

These two properties can be obtained in a perfect, statistical or computational way, according to the power an adversary would need to break them. But essentially, a perfectly binding commitment scheme guarantees the uniqueness of the opening phase. This is achieved by an encryption scheme, which on the other hand provides the computational hiding property only, under the IND-CPA security. A perfectly hiding commitment scheme guarantees the perfect secrecy of $m$.

Some additional properties are sometimes required. The first one ie extractability, for a perfectly binding commitment scheme. The latter admits an indistinguishable Setup phase that also generates a trapdoor allowing message extraction from the commitment. Again, an encryption scheme is an extractable commitment, where the decryption key is the trapdoor that allows extraction. The second one is equivocability, for a perfectly hiding commitment scheme. The latter admits an indistinguishable Setup phase that generates a trapdoor allowing to open a commitment in any way.

\subsection{Universal Composability Framework}

The Universal Composability framework was introduced in [Can01]. The aim of the following is just to give a brief overview to have some common conventions.

In the context of multi-party computation, one wants several users $P_{i}$ with inputs $x_{i}$ to be able to compute a specific function $f\left(x_{1}, \ldots, x_{n}\right)=\left(y_{1}, \ldots, y_{n}\right)$ without leaking anything except $y_{i}$ to $P_{i}$. One

\begin{tabular}{|c|c|c|c|c|c|c|c|c|}
\hline Scheme & \multicolumn{3}{|c|}{$\begin{array}{c}\text { Communication } \\
\text { Complexity (in bits) } \\
\text { Commit Decommit Total }\end{array}$} & \multicolumn{2}{|c|}{$\begin{array}{c}\text { Round } \\
\text { Complexity } \\
\text { Commit Decommit }\end{array}$} & \multicolumn{2}{|c|}{$\begin{array}{l}\text { Computation } \\
\text { Complexity } \\
\text { exp. pair. }\end{array}$} & Adaptivity \\
\hline$[\operatorname{Lin} 11 \mathrm{a}, \S 3]$ & 1024 & 2560 & 3584 & 1 & 5 & 27 & - & $x$ \\
\hline$[\operatorname{Lin} 11 \mathrm{a}, \S 4]$ & 3072 & 1536 & 4608 & 5 & 1 & 36 & - & $\checkmark$ \\
\hline [FLM11, $\S 3]$ & 2560 & 8192 & 10752 & 1 & 1 & 41 & $69^{2}$ & $\checkmark$ \\
\hline$[$ FLM11, $\S 4]$ & 18944 & 1536 & 20480 & 1 & 1 & 88 & $129^{2}$ & $\checkmark$ \\
\hline Fig. 6 & 1024 & 2048 & 3072 & 1 & 3 & 22 & - & $x$ \\
\hline Fig. 7 & 2048 & 1536 & 3584 & 3 & 1 & 26 & - & $\checkmark$ \\
\hline
\end{tabular}

Table 1. Efficiency comparison of UC-secure commitment schemes (128-bit security) 
$\mathcal{F}_{\text {mcom }}$ with session identifier sid proceeds as follows, running with parties $P_{1}, \ldots, P_{n}$, a parameter $1^{\mathfrak{K}}$, and an adversary $\mathcal{S}$ :

- Commit phase: Upon receiving a message (Commit, sid, ssid, $\left.P_{i}, P_{j}, x\right)$ from $P_{i}$ where $x \in\{0,1\}^{\text {polylogk}}$, record the tuple (ssid, $\left.P_{i}, P_{j}, x\right)$ and send the message (receipt, sid, ssid, $\left.P_{i}, P_{j}\right)$ to $P_{j}$ and $\mathcal{S}$.

- Reveal phase: Upon receiving a message of the form (reveal, sid, ssid) from party $P_{i}$, if a tuple (ssid, $\left.P_{i}, P_{j}, m\right)$ was previously recorded, then send the message (reveal, sid, ssid, $\left.P_{i}, P_{j}, x\right)$ to $P_{j}$ and $\mathcal{S}$. Otherwise, ignore.

Fig. 1. Ideal Functionality $\mathcal{F}_{\text {mcom }}$ for Commitment

can think about Yao's Millionaires' problem [Yao82]. Instead of following the classical approach which aims at listing exhaustively all the expected properties, Canetti did something else and tried to define how a protocol should ideally work: what are the inputs, and what are the available outputs. For that, he specified two worlds: the real world, where the protocol is run with some possible attacks, and the ideal world where everything would go smoothly, and namely no damage can be done with the protocol. For a good protocol instantiation, it should be impossible to distinguish, for an external player, the real world from the ideal one.

In the ideal world there is indeed an incorruptible entity named the ideal functionality, to which players can send their inputs privately, and then receive the corresponding outputs without any kind of communication between the players. This way the functionality can be set to be correct, without revealing anything except what is expected. It is thus perfectly secure. A protocol, in the real world with real players and thus possibly malicious players, should create executions that look similar to the ones in the previous world. This is to show that the communication between the players should not give more information than the functionality's description and its outputs.

As a consequence, the formal security proof is performed by showing that for any external entity, that gives inputs to the honest players and gets the outputs but that also controls the adversary, the executions in the two above worlds are indistinguishable. More concretely, in order to prove that a protocol $\mathcal{P}$ realizes an ideal functionality $\mathcal{F}$, we consider an environment $\mathcal{Z}$ which can choose inputs given to all the honest players and receives back the outputs they get, but which also controls an adversary $\mathcal{A}$. Its goal is to distinguish in which case it is: either the real world with concrete interactions between the players and the adversary, or the ideal world in which players simply forward everything to and from the ideal functionality and the adversary interacts with a simulator $\mathcal{S}$ to attack the ideal functionality. We have to build a simulator $\mathcal{S}$ that makes the two views indistinguishable to the environment: since the combination of the adversary and the simulator cannot cause any damage against the ideal functionality, this shows that the adversary cannot cause any damage either against the real protocol.

The main constraint is that the simulator cannot rewind the execution as often done in classical proofs, since it interacts with an adversary under the control of the environment: there is no possible rewind in the real word, it is thus impossible too in the ideal world.

The adversary $\mathcal{A}$ has access to the communication but nothing else, and namely not to the inputs/ouputs for the honest players. In case of corruption, it gets complete access to inputs and the internal memory of the honest player, and then gets control of it.

\subsection{Ideal Functionality for Commitments}

A UC-secure commitment scheme provides all the properties previously given: it should be hiding and binding, but also extractable and equivocable, and even non-malleable. The ideal functionality is presented on Figure 1. It is borrowed from [Lin11a].

\subsection{Useful Primitives}

Hash Function Family. A hash function family $\mathcal{H}$ is a family of functions $\mathfrak{H}_{K}$ from $\{0,1\}^{*}$ onto a fix-length output, either $\{0,1\}^{k}$ or $\mathbb{Z}_{p}$. 
Definition 1 (Universal One-Way [NY89]). A family is said to be universal one-way if for any adversary $\mathcal{A}$ on a family $\mathcal{H}$, it should be hard for it to conveniently pick a scalar $x$, such that it is able to find a second-preimage for a random function $\mathfrak{H}_{K} \in \mathcal{H}$. More precisely, we denote

$$
\begin{aligned}
\operatorname{Succ}_{\mathcal{H}}^{\text {uow }}(\mathcal{A}) & =\operatorname{Pr}\left[x \leftarrow \mathcal{A}(\mathcal{H}), \mathfrak{H}_{K} \stackrel{\$}{\leftarrow} \mathcal{H}, y \leftarrow \mathcal{A}\left(\mathfrak{H}_{K}\right): \mathfrak{H}_{K}(x)=\mathfrak{H}_{K}(y)\right] \\
\operatorname{Succ}_{\mathcal{H}}^{\text {uow }}(t) & =\max _{\mathcal{A} \leq t}\left\{\operatorname{Succ}_{\mathcal{H}}^{\text {uow }}(\mathcal{A})\right\}
\end{aligned}
$$

In some cases, we may rely on a stronger property when we cannot postpone the choice of the hash function in our simulations:

Definition 2 (Collision-Resistant). A family is said to be collision-resistant if for any adversary $\mathcal{A}$ on a random function $\mathfrak{H}_{K} \stackrel{\$}{\leftarrow} \mathcal{H}$, it is hard to find a collision. More precisely, we denote

$$
\begin{aligned}
\operatorname{Succ}_{\mathcal{H}}^{\text {coll }}(\mathcal{A}) & =\operatorname{Pr}\left[\mathfrak{H}_{K} \stackrel{\$}{\leftarrow} \mathcal{H},\left(m_{0}, m_{1}\right) \leftarrow \mathcal{A}\left(\mathfrak{H}_{K}\right): \mathfrak{H}_{K}\left(m_{0}\right)=\mathfrak{H}_{K}\left(m_{1}\right)\right] \\
\operatorname{Succ}_{\mathcal{H}}^{\text {coll }}(t) & =\max _{\mathcal{A} \leq t}\left\{\operatorname{Succ}_{\mathcal{H}}^{\text {coll }}(\mathcal{A})\right\} .
\end{aligned}
$$

Pedersen Commitment. The Pedersen commitment [Ped92] is an equivocable commitment:

- Setup $\left(1^{\mathfrak{K}}\right)$ generates a group $\mathbb{G}$ of order $p$, with two independent generators $g$ and $\zeta$;

- Commit $(m ; r)$, for a message $m \stackrel{\$}{\leftarrow} \mathbb{Z}_{p}$ and random coins $r \stackrel{\$}{\leftarrow} \mathbb{Z}_{p}$, produces a commitment $c=$ $\operatorname{Ped}(m, r)=g^{m} \zeta^{r}$, while $r$ is the opening information;

- Decommit $(c, m ; r)$ outputs $m$ and $r$, which opens $c$ into $m$, and allows the validity test $c \stackrel{?}{=} g^{m} \zeta^{r}$.

This commitment is computationally binding under the discrete logarithm assumption: two different openings $(m, r)$ and $\left(m^{\prime}, r^{\prime}\right)$ for a commitment $c$, lead to the discrete logarithm of $\zeta$ in basis $g$. On the other hand, with this discrete logarithm value as additional information from the setup, one can equivocate any dummy commitment, when the input and opening values are known.

Cramer-Shoup Encryption. The Cramer-Shoup encryption scheme [CS98] is an IND-CCA version of the ElGamal encryption. By merging the Setup and KeyGen algorithm into a unique Setup algorithm, we make it into an extractable commitment scheme CS, where $\mathrm{dk}$ is the extraction key, and $r$ is the witness for the opening.

- $\operatorname{Setup}\left(1^{\mathfrak{K}}\right)$ generates a group $\mathbb{G}$ of order $p$.

- KeyGen(param) generates $\left(g_{1}, g_{2}\right) \stackrel{\$}{\leftarrow} \mathbb{G}^{2}, \mathrm{dk}=\left(x_{1}, x_{2}, y_{1}, y_{2}, z\right) \stackrel{\$}{\leftarrow} \mathbb{Z}_{p}^{5}$, and sets $c=g_{1}^{x_{1}} g_{2}^{x_{2}}, d=g_{1}^{y_{1}} g_{2}^{y_{2}}$, and $h=g_{1}^{z}$. It also chooses a Collision-Resistant hash function $\mathfrak{H}_{K}$ in a hash family $\mathcal{H}$ (or a Universal One-Way Hash Function). The encryption key is ek $=\left(g_{1}, g_{2}, c, d, h, \mathfrak{H}_{K}\right)$.

- Encrypt(ek, $M ; r)$, for a message $M \in \mathbb{G}$ and a random scalar $r \in \mathbb{Z}_{p}$, the ciphertext is $C=\operatorname{CS}(M ; r)=$ $\left(\mathbf{u}=\left(g_{1}^{r}, g_{2}^{r}\right), e=M \cdot h^{r}, v=\left(c d^{\omega}\right)^{r}\right)$, where $v$ is computed afterwards with $\omega=\mathfrak{H}_{K}(\mathbf{u}, e)$.

- Decrypt $(\mathrm{dk}, C)$ : one first computes $\omega=\mathfrak{H}_{K}(\mathbf{u}, e)$ and checks whether $u_{1}^{x_{1}+\omega y_{1}} \cdot u_{2}^{x_{2}+\omega y_{2}} \stackrel{?}{=} v$ or not. If the equality holds, one computes $M=e /\left(u_{1}^{z}\right)$ and outputs $M$. Otherwise, one outputs $\perp$.

The IND-CCA security can be proven under the DDH assumption and the fact the hash function used is a Universal One-Way Hash Function. This also leads to a non-malleable commitment scheme, that is additionally extractable when the Setup outputs the decryption key dk.

\section{Lindell's Commitment Protocols}

We now have all the tools to review the two original Lindell's commitment schemes [Lin11a]. 
We have a CRS, consisting of $\left(p, \mathbb{G}, g_{1}, g_{2}, c, d, h, h_{1}, h_{2}\right)$, where $\mathbb{G}$ is a group of order $p$ with generators $g_{1}, g_{2} ; c, d, h \in \mathbb{G}$ are random elements in $\mathbb{G}$ and $h_{1}=g_{1}{ }^{\rho}$ and $h_{2}=g_{2}{ }^{\rho}$ for a random $\rho \in \mathbb{Z}_{p}$.

Intuitively, $\left(p, \mathbb{G}, g_{1}, g_{2}, c, d, h\right)$ is a Cramer-Shoup encryption key and $\left(p, \mathbb{G}, g_{1}, g_{2}, h_{1}, h_{2}\right)$ is the CRS of a dual-mode encryption scheme.

Let $G:\{0,1\}^{n} \rightarrow \mathbb{G}$ be an efficiently computable and invertible mapping of a binary string to the group.

The commit phase

Upon receiving a message (Commit, sid, ssid, $\left.P_{i}, P_{j}, x\right)$ where $x \in\{0,1\}^{n-\log ^{2}(n)}$ and sid, ssid $\in\{0,1\}^{\log ^{2}(n) / 4}$, party $P_{i}$ works as follows:

1. $P_{i}$ computes $m=G\left(x\right.$, sid, ssid, $\left.P_{i}, P_{j}\right)$.

2. $P_{i}$ picks $r \stackrel{\$}{\leftarrow} \mathbb{Z}_{p}$ and computes $C=\mathrm{CS}(m ; r)$, we will note $\omega$ the hash of the first three terms.

3. $P_{i}$ sends (sid, ssid, $C$ ) to $P_{j}$.

4. $P_{j}$ stores (sid, ssid, $\left.P_{i}, P_{j}, c\right)$ and outputs (receipt, sid, ssid, $P_{i}, P_{j}$ ).

$P_{j}$ ignores any later commitment messages with the same (sid, ssid) from $P_{i}$.

The decommit phase

Upon receiving a message (reveal, sid, ssid, $\left.P_{i}, P_{j}\right), P_{i}$ works as follows:

1. $P_{i}$ sends (sid, ssid, $x$ ) to $P_{j}$.

2. $P_{j}$ computes $m=G\left(x\right.$, sid, ssid, $\left.P_{i}, P_{j}\right)$

3. (a) $P_{j}$ picks $R, S \stackrel{\$}{\leftarrow} \mathbb{Z}_{p}$, a random challenge $\varepsilon \stackrel{\$}{\leftarrow}\{0,1\}^{n}$ and sends $c^{\prime}=\left(g_{1}{ }^{R} g_{2}{ }^{S}, h_{1}{ }^{r} h_{2}{ }^{S} G(\varepsilon)\right)$ to $P_{i}$.

(b) $P_{i}$ picks $s \stackrel{\$}{\leftarrow} \mathbb{Z}_{p}$ and computes $(\alpha, \beta, \gamma, \delta)=\left(g_{1}{ }^{s}, g_{2}{ }^{s}, h^{s},\left(c d^{\omega}\right)^{s}\right)$.

He then sends (sid, ssid, $\alpha, \beta, \gamma, \delta$ ) to $P_{j}$.

(c) $P_{j}$ now opens $c^{\prime}$ by sending (sid, ssid, $R, S, \varepsilon$ ) to $P_{i}$.

(d) $P_{i}$ checks if this is consistent with $c^{\prime}$ otherwise he aborts. $P_{i}$ now computes $z=s+\varepsilon r$ and sends (sid, ssid, $z$ ) to $P_{j}$.

(e) $P_{j}$ outputs (reveal, sid, ssid, $\left.P_{i}, P_{j}, x\right)$ if and only if

$$
g_{1}^{z}=\alpha u_{1}{ }^{\varepsilon}, g_{2}{ }^{z}=\beta u_{2}{ }^{\varepsilon}, h^{z}=\gamma(e / m)^{\varepsilon},\left(c d^{\omega}\right)^{z}=\delta v^{\varepsilon}
$$

Fig. 2. Lindell's Commitment Protocol, UC-Secure against Static Corruptions

\subsection{Static Corruptions}

The first variant, presented on Figure 2 only prevents static corruptions: the adversary can decide to run the protocol on behalf of a player, with its inputs, from the beginning, but cannot corrupt anybody when the execution has started.

\subsection{Adaptive Corruptions}

The second variant prevents adaptive corruptions. It is presented on Figure 3. The main difference from the previous scheme is to move some proof from the decommit phase to the commit phase.

\section{Discussion and Correction}

\subsection{Discussion}

Adaptive Corruptions. Lindell has proven both schemes secure under the DDH assumption, the former in details but a sketch of proof only for the latter. And actually, as noted by Lindell in the last version of [Lin11b], the security against adaptive corruptions might eventually not be guaranteed.

He indeed proves that no adversary can choose a message $x^{\prime}$ beforehand, and do a valid commit/decommit sequence to $x^{\prime}$ where the simulator extraction, at the end of the commit phase, would output an $x$ different from $x^{\prime}$. However this is not enough as an adversary could still do a valid commit/decommit sequence to $x^{\prime}$ where the simulator extraction at the end of the commit phase would output an $x$ different from $x^{\prime}$. The difference between the two experiments is how much the adversary 
We have a CRS, consisting of $\left(p, \mathbb{G}, g_{1}, g_{2}, c, d, h, h_{1}, h_{2}, \zeta, \mathfrak{H}_{K}\right)$, where $\mathbb{G}$ is a group of order $p$ with generators $g_{1}, g_{2}$; $c, d, h \in \mathbb{G}$ are random elements in $\mathbb{G}$ and $h_{1}=g_{1}{ }^{\rho}$ and $h_{2}=g_{2}{ }^{\rho}$ for a random $\rho \in \mathbb{Z}_{p} ; \mathfrak{H}_{K}$ is randomly drawn from a collision-resistant hash function family $\mathcal{H}$.

Intuitively $\left(p, \mathbb{G}, g_{1}, g_{2}, c, d, h, \mathfrak{H}_{K}\right)$ is a Cramer-Shoup encryption key, $\left(p, \mathbb{G}, g_{1}, g_{2}, h_{1}, h_{2}\right)$ is the CRS of a dual-mode encryption scheme, and $(p, \mathbb{G}, g, \zeta)=$ is the CRS of a Pedersen commitment scheme.

Let $G:\{0,1\}^{n} \rightarrow \mathbb{G}$ be an efficiently computable and invertible mapping of a binary string to the group.

The commit phase

Upon receiving a message (Commit, sid, ssid, $\left.P_{i}, P_{j}, x\right)$ where $x \in\{0,1\}^{n-\log ^{2}(n)}$ and sid, ssid $\in\{0,1\}^{\log ^{2}(n) / 4}$, party $P_{i}$ works as follows:

1. $P_{i}$ computes $m=G\left(x\right.$, sid, ssid, $\left.P_{i}, P_{j}\right)$.

2. $P_{i}$ picks $r \stackrel{\$}{\leftarrow} \mathbb{Z}_{p}$ and computes $C=\mathrm{CS}(m ; r)$, we will note $\omega$ the hash of the first three terms.

3. $P_{i}$ picks $k_{1} \stackrel{\$}{\leftarrow} \mathbb{Z}_{p}$, computes $c_{p}^{1}=\operatorname{Ped}\left(\mathfrak{H}_{K}(C) ; k_{1}\right)$ and sends it to $P_{j}$.

4. $P_{j}$ picks $R, S \stackrel{\$}{\leftarrow} \mathbb{Z}_{p}, \varepsilon \stackrel{\$}{\leftarrow}\{0,1\}^{n}$ and sends $c^{\prime}=\left(g_{1}{ }^{R} g_{2}{ }^{S}, h_{1}{ }^{R} h_{2}{ }^{S} G(\varepsilon)\right)$ to $P_{i}$.

5. $P_{i}$ picks $s, k_{2} \stackrel{\$}{\leftarrow} \mathbb{Z}_{p}$ and computes $(\alpha, \beta, \gamma, \delta)=\left(g_{1}{ }^{s}, g_{2}{ }^{s}, h^{s},\left(c d^{\omega}\right)^{s}\right)$.

He then computes and sends $c_{p}^{2}=\operatorname{Ped}\left(\mathfrak{H}_{K}(\alpha, \beta, \gamma, \delta) ; k_{2}\right)$ to $P_{j}$.

6. $P_{j}$ now opens $c^{\prime}$ by sending $(R, S, \varepsilon)$ to $P_{i}$.

7. $P_{i}$ checks if this is consistent with $c^{\prime}$ otherwise he aborts.

8. $P_{i}$ now computes $z=s+\varepsilon r$, and erases $r, s$.

He also opens $c_{p}^{1}$ by sending $C, k_{1}$ to $P_{j}$.

9. $P_{j}$ verifies the consistency of $c_{p}^{1}$.

If yes, he stores (sid, ssid, $P_{i}, P_{j}, c, \varepsilon, c_{p}^{2}$ ) and outputs (receipt, sid, ssid, $P_{i}, P_{j}$ ).

He ignores any later commitment messages with the same (sid, ssid) from $P_{i}$.

The decommit phase

Upon receiving a message (reveal, sid, ssid, $\left.P_{i}, P_{j}\right), P_{i}$ works as follows:

1. $P_{i}$ sends $\left(x, \alpha, \beta, \gamma, \delta, k_{2}, z\right)$ to $P_{j}$.

2. $P_{j}$ computes $m=G\left(x\right.$, sid, ssid, $\left.P_{i}, P_{j}\right)$, and outputs (reveal, sid, ssid, $\left.P_{i}, P_{j}, x\right)$ if and only if $c_{p}^{2}$ is consistent and:

$$
g_{1}^{z}=\alpha u_{1}^{\varepsilon}, g_{2}^{z}=\beta u_{2}{ }^{\varepsilon}, h^{z}=\gamma(e / m)^{\varepsilon},\left(c d^{\omega}\right)^{z}=\delta v^{\varepsilon}
$$

Fig. 3. Lindell's Commitment Protocol, UC-Secure against Adaptive Corruptions with Erasures

controls the value $x^{\prime}$ : in the former $x^{\prime}$ has to be chosen beforehand, while in the latter $x^{\prime}$ is any value different from $x$.

We describe, on Figure 4, such a situation in which the adversary $\mathcal{A}$ plays as $P_{i}$, and makes the simulator extract the value $x$, while in fact committing (or actually opening) to another value $x^{\prime}$. For the sake of clarity, we only mention the differences between this situation and the real protocol presented on Figuknyextraction done on $C$ at the end of the commit phase would lead the simulator to believe to a commit to $x$, however the valid decommit outputs $x^{\prime}$. Note however that this attack does not succeed very often since one needs, for a random $\varepsilon$, that $G^{-1}\left(M D^{1 / \varepsilon}\right)$ exists and can be parsed as $\left(x^{\prime}\right.$, sid, ssid, $\left.P_{i}, P_{j}\right)$.

Static Corruptions. We stress that this possible inconsistency comes from the move forward of the proof in the commit phase, even before the message $x$ is strongly committed. The first protocol does not suffer from this issue.

\subsection{A Simple Patch}

In order to avoid the above concern, a simple patch consists in committing $m=G\left(x\right.$, sid, ssid, $\left.P_{i}, P_{j}\right)$ in the second Pedersen commitment $c_{p}^{2}$. This leads to the simple change in the protocol presented on Figure 5, where $x$ is now strongly committed before the proof, and then the previous issue does not occur anymore.

We do not give more details about this proof, since we will now focus on much more efficient protocols, with the above modification, and additional ones. 


\section{The commit phase}

Upon receiving a message (Commit, sid, ssid, $\left.P_{i}, P_{j}, x\right)$ where $x \in\{0,1\}^{n-\log ^{2}(n)}$ and sid, ssid $\in\{0,1\}^{\log ^{2}(n) / 4}, \mathcal{A}$ works as follows:

5. $\mathcal{A}$ picks $s, k_{2} \stackrel{\$}{\leftarrow} \mathbb{Z}_{p}, \underline{D} \stackrel{\$}{\leftarrow} \mathbb{G}$ and computes $(\alpha, \beta, \gamma, \delta)=\left(g_{1}{ }^{s}, g_{2}{ }^{s}, h^{s} \underline{D},\left(c d^{\omega}\right)^{s}\right)$. $\mathcal{A}$ then computes and sends $c_{p}^{2}=\operatorname{Ped}\left(\mathfrak{H}_{K}(\alpha, \beta, \gamma, \delta) ; k_{2}\right)$ to $P_{j}$.

8. $\mathcal{A}$ checks if $G^{-1}\left(M D^{1 / \varepsilon}\right)$ exists and can be parsed as $\left(x^{\prime}\right.$, sid, ssid, $\left.P_{i}, P_{j}\right)$ for a random $x^{\prime}$.

If so, $\mathcal{A}$ now computes $z=s+\varepsilon r$, and erases $r, s$.

It also opens $c_{p}^{1}$ by sending $C, k_{1}$ to $P_{j}$.

The decommit phase

Upon receiving a message (reveal, sid, ssid, $\left.P_{i}, P_{j}\right), \mathcal{A}$ works as follows:

1. $\mathcal{A}$ sends $\left(\underline{x^{\prime}}, \alpha, \beta, \gamma, \delta, k_{2}, z\right)$ to $P_{j}$.

2. $P_{j}$ computes $\underline{m^{\prime}}=G\left(\right.$ sid, ssid, $\left.P_{i}, P_{j}, \underline{x}\right)$, and outputs (reveal, sid, ssid, $P_{i}, P_{j}, \underline{x^{\prime}}$ ) if and only if $c_{p}^{2}$ is consistent and:

$$
g_{1}^{z}=\alpha u_{1}^{\varepsilon}, g_{2}^{z}=\beta u_{2}^{\varepsilon}, h^{z}=\underline{h^{s}\left(e^{\prime} D^{1 / \varepsilon}\right)^{\varepsilon}}=\gamma\left(e / \underline{m^{\prime}}\right)^{\varepsilon},\left(c d^{\omega}\right)^{z}=\delta v^{\varepsilon}
$$

Fig. 4. Inconsistent Extraction and Opening with the Protocol from Figure 3

\section{Our Optimization of the Commitments Protocols}

We kept the original notations, but as done in $\left[\mathrm{BBC}^{+} 13\right]$, we can note that $C$ is actually a Cramer-Shoup encryption of $m$, and $(\alpha, \beta, \gamma, \delta)$ is a partial Cramer-Shoup encryption of 1 with the same $\omega$ as in the first ciphertext: the double Cramer-Shoup encryption of $\left(m, m^{\prime}\right)$ was denoted by $\operatorname{DCS}\left(m, m^{\prime} ; r, s\right)=\left(C_{1}, C_{2}\right)$, where

- $C_{1}$ is a real Cramer-Shoup encryption $C_{1}=\operatorname{CS}(m ; r)$ of $m$ for a random $r \stackrel{\$}{\leftarrow} \mathbb{Z}_{p}$ : $C_{1}=\left(\mathbf{u}_{1}=\right.$ $\left.\left(g_{1}{ }^{r}, g_{2}{ }^{r}\right), e_{1}=m \cdot h^{r}, v_{1}=\left(c d^{\omega}\right)^{r}\right)$, where $v_{1}$ is computed afterwards with $\omega=\mathfrak{H}_{K}\left(\mathbf{u}_{1}, e_{1}\right)$;

- $C_{2}$ is a partial Cramer-Shoup encryption $C_{2}=\operatorname{PCS}\left(m^{\prime} ; \omega, s\right)$ of $m^{\prime}$ for a random $s \stackrel{\$}{\leftarrow} \mathbb{Z}_{p}$ with the above $\omega$ value: $C_{2}=\left(\mathbf{u}_{2}=\left(g_{1}{ }^{s}, g_{2}{ }^{s}\right), e_{2}=m^{\prime} \cdot h^{s}, v_{2}=\left(c d^{\omega}\right)^{s}\right)$, where $v_{2}$ is computed directly with the above $\omega=\mathfrak{H}_{K}\left(\mathbf{u}_{1}, e_{1}\right)$.

In addition, when $\omega$ is fixed, we have an homomorphic property: if $\left(C_{1}, C_{2}\right)=\operatorname{DCS}\left(m, m^{\prime} ; r, s\right)$, with a common $\omega$, the component-wise product $C_{1} \times C_{2}=\operatorname{PCS}\left(m \times m^{\prime} ; \omega, r+s\right)$. In particular, we can see the last tuple $\left(\alpha u_{1}^{\varepsilon}, \beta u_{2}^{\varepsilon}, \gamma e^{\varepsilon}, \delta v^{\varepsilon}\right)$ as $C_{2} \times C_{1}^{\varepsilon}$. It should thus be $\operatorname{PCS}\left(m^{\varepsilon} ; \omega, \varepsilon r+s\right)=\operatorname{PCS}\left(m^{\varepsilon} ; \omega, z\right)$, which is the final check. We now use these new notations in the following.

\subsection{Improvement of the Static Protocol}

The improvement presented below consists in noting that the receiver can directly send the value $\varepsilon$ in the decommit phase, without having to send a commitment first. To allow this, we simply ask the sender to send a Pedersen commitment of $C_{2}=(\alpha, \beta, \gamma, \delta)$ prior to receiving $\varepsilon$. This reduces the number of flows of the decommit phase (from 5 downto 3 ) and the number of elements sent by the receiver, (from 2 group elements and 3 scalars down to only 1 scalar, the challenge), simply increasing the number of elements sent by the sender by 1 group element and 1 scalar (the Pedersen commitment).

\section{The commit phase}

5. $P_{i}$ picks $s, k_{2} \stackrel{\$}{\leftarrow} \mathbb{Z}_{p}$ and computes $(\alpha, \beta, \gamma, \delta)=\left(g_{1}{ }^{s}, g_{2}{ }^{s}, h^{s},\left(c d^{\omega}\right)^{s}\right)$. He then computes and sends $c_{p}^{2}=\operatorname{Ped}\left(\underline{m}, \mathfrak{H}_{K}(\alpha, \beta, \gamma, \delta) ; k_{2}\right)$ to $P_{j}$.

Fig. 5. Simple Patch to the Protocol from Figure 3 
We have a CRS, consisting of $\left(p, \mathbb{G}, g, g_{1}, g_{2}, c, d, h, \zeta, \mathfrak{H}_{K}\right)$, where $\mathbb{G}$ is a group of order $p$ with generators $g, \zeta, g_{1}, g_{2} ;$ $c, d, h \in \mathbb{G}$ are random elements in $\mathbb{G} ; \mathfrak{H}_{K}$ is randomly drawn from a collision-resistant hash function family $\mathcal{H}$.

Intuitively $\left(p, \mathbb{G}, g_{1}, g_{2}, c, d, h, \mathfrak{H}_{K}\right)$ is a Cramer-Shoup public key and $(p, \mathbb{G}, g, \zeta)$ is a CRS for a Pedersen commitment.

Let $G:\{0,1\}^{n} \rightarrow \mathbb{G}$ be an efficiently computable and invertible mapping of a binary string to the group, as before.

The commit phase

Upon receiving a message (Commit, sid, ssid, $\left.P_{i}, P_{j}, x\right)$ where $x \in\{0,1\}^{n-\log ^{2}(n)}$ and sid, ssid $\in\{0,1\}^{\log ^{2}(n) / 4}$, party $P_{i}$ works as follows:

1. $P_{i}$ computes $m=G\left(x\right.$, sid, ssid, $\left.P_{i}, P_{j}\right)$.

2. $P_{i}$ picks $r, s \stackrel{\$}{\leftarrow} \mathbb{Z}_{p}$ and computes $\left(C_{1}, C_{2}\right)=\operatorname{DCS}(m, 1 ; r, s)$.

We note $C_{2}=(\alpha, \beta, \gamma, \delta)$.

3. $P_{i}$ sends (sid, ssid, $C_{1}$ ) to $P_{j}$.

4. $P_{j}$ stores (sid, ssid, $\left.P_{i}, P_{j}, C_{1}\right)$ and outputs (receipt, sid, ssid, $P_{i}, P_{j}$ ).

He ignores any later commitment messages with the same (sid, ssid) from $P_{i}$.

The decommit phase

Upon receiving a message (reveal, sid, ssid, $\left.P_{i}, P_{j}\right), P_{i}$ works as follows:

1. $P_{i}$ picks $k_{2} \stackrel{\$}{\leftarrow} \mathbb{Z}_{p}$, computes $c_{p}^{2}=\operatorname{Ped}\left(\mathfrak{H}_{K}\left(m, C_{2}\right.\right.$, sid, ssid, $\left.\left.P_{i}, P_{j}\right) ; k_{2}\right)$ and sends (sid, ssid, $\left.x, c_{p}^{2}\right)$ to $P_{j}$.

2. $P_{j}$ computes $m=G\left(x\right.$, sid, ssid, $\left.P_{i}, P_{j}\right)$, picks $\varepsilon \stackrel{\$}{\leftarrow} \mathbb{Z}_{p}$ and sends it to $P_{i}$.

3. $P_{i}$ now computes $z=s+\varepsilon r$ and sends (sid, ssid, $C_{2}, k_{2}, z$ ) to $P_{j}$.

4. $P_{j}$ outputs (reveal, sid, ssid, $\left.P_{i}, P_{j}, x\right)$ if and only if $c_{p}^{2}$ is consistent and

$$
g_{1}^{z}=\alpha u_{1}^{\varepsilon}, g_{2}^{z}=\beta u_{2}{ }^{\varepsilon}, h^{z}=\gamma(e / m)^{\varepsilon},\left(c d^{\omega}\right)^{z}=\delta v^{\varepsilon}
$$

Fig. 6. Our New Static UC-Secure Commitment Protocol

\subsection{Sketch of Proof of the Static Protocol}

For lack of space, we do not give here the full proof of the protocol. One may note that it is very similar to the one given in [Lin11a]. The only change lies in the decommit phase, where we make the receiver directly send his challenge value $\varepsilon$ rather than encrypting it first. But this change is made possible by the sender sending a Pedersen commitment $c_{p}^{2}$ of $C_{2}$ before having seen $\varepsilon$, as in the commit phase of the adaptive version of our protocol.

The proof can thus be easily adapted from the one given for our adaptive protocol (see Section 5.4). The only difference is that in the static version, the sender does not commit to his value $C_{1}$, so that the simulator cannot change its mind on the value it gave inside this ciphertext later on. But one can note that in the proof of the adaptive protocol, this commitment $c_{p}^{1}$ has to be equivocated only in case of adaptive corruptions (if the latter occur before the adversary has sent $\varepsilon$ ). This yields to the same simulator as in the adaptive case (see Section 5.5) with the following modifications, when $P_{i}$ is honest only:

Commit stage: Exactly as in the adaptive case except there is no corruption to deal with.

DeCommit STAGE: Upon receiving the information that the decommitment has been performed on $x$, with (reveal, sid, ssid, $\left.P_{i}, P_{j}, x\right)$ from $\mathcal{F}_{\text {mcom }}, \mathcal{S}$ first chooses a random $z$ and computes the ciphertext $C_{3}=$ $\operatorname{PCS}(m ; \omega, z)$. It then chooses a random $k_{2}$, a random $C_{2}$, computes the associated Pedersen commitment $c_{p}^{2}$ and simulates the first flow of the decommit phase to $P_{j}$. Upon receiving $\varepsilon$ from $P_{j}$, it then adapts $C_{2}=C_{3} / C_{1}{ }^{\varepsilon}$ and uses the trapdoor for the Pedersen commitment to produce a new value $k_{2}$ corresponding to the new value $C_{2}$. It then simulates the third flow of the decommit phase to $P_{j}$.

\subsection{Improvement of the Adaptive Protocol}

As for the static version of the protocol, the main improvement presented in the figure 7 below consists in noting that the receiver can directly send the value $\varepsilon$, without having to send an encryption before. To allow this, we simply ask the sender to send his two Pedersen commitments prior to receiving $\varepsilon$.

This reduces, in the commit phase, the number of rounds (from 5 downto 3) and the number of elements sent by the receiver (from 2 group elements and 3 scalars down to only 1 scalar, the challenge). 
Contrary to the static version, there is no additional cost. This is illustrated in Figure 8, which sums up the differences between Lindell's protocol and ours, in the same setting: UC-security against adaptive corruption with erasures.

In addition, in order to slightly increase the message space from $n-\log _{2}(n)$ to $n$, we move the sensitive prefix (sid, ssid, $P_{i}, P_{j}$ ) into the second Pedersen.

Eventually, in order to definitely exclude the security concerns presented in Section 4, we include the value $m$ to the second Pedersen to prevent the adversary from trying to open his commitment to another value.

We have a CRS, consisting of $\left(p, \mathbb{G}, g, g_{1}, g_{2}, c, d, h, \zeta, \mathfrak{H}_{K}\right)$, where $\mathbb{G}$ is a group of order $p$ with generators $g, \zeta, g_{1}, g_{2} ;$ $c, d, h \in \mathbb{G}$ are random elements in $\mathbb{G} ; \mathfrak{H}_{K}$ is randomly drawn from a collision-resistant hash function family $\mathcal{H}$.

Intuitively $\left(p, \mathbb{G}, g_{1}, g_{2}, c, d, h, \mathfrak{H}_{K}\right)$ is a Cramer-Shoup public key and $(p, \mathbb{G}, g, \zeta)$ is a CRS for a Pedersen commitment.

Let $G:\{0,1\}^{n} \rightarrow \mathbb{G}$ be an efficiently computable and invertible mapping of a binary string to the group, as before.

The commit phase

Upon receiving a message (Commit, sid, ssid, $\left.P_{i}, P_{j}, x\right)$, party $P_{i}$ works as follows, where $x \in\{0,1\}^{n}$ and sid, ssid $\in$ $\{0,1\}^{\log ^{2}(n) / 4}$ :

1. $P_{i}$ computes $m=G(x)$.

2. $P_{i}$ picks $r, s \stackrel{\$}{\leftarrow} \mathbb{Z}_{p}$ and computes $\left(C_{1}, C_{2}\right)=\operatorname{DCS}(m, 1 ; r, s)$.

We note $C_{2}=(\alpha, \beta, \gamma, \delta)$.

3. $P_{i}$ picks $k_{1}, k_{2} \stackrel{\$}{\leftarrow} \mathbb{Z}_{p}$.

He computes $c_{p}^{1}=\operatorname{Ped}\left(\mathfrak{H}_{K}\left(C_{1}\right) ; k_{1}\right), c_{p}^{2}=\operatorname{Ped}\left(\mathfrak{H}_{K}\left(m, C_{2}\right.\right.$, sid, ssid, $\left.\left.P_{i}, P_{j}\right) ; k_{2}\right)$.

He sends $\left(c_{p}^{1}, c_{p}^{2}\right)$ to $P_{j}$.

4. $P_{j}$ picks $\varepsilon \stackrel{\$}{\leftarrow} \mathbb{Z}_{p}$ and sends it to $P_{i}$.

5. $P_{i}$ now computes $z=s+\varepsilon r$, and erases $r, s$.

He also opens $c_{p}^{1}$ by sending $\left(C_{1}, k_{1}\right)$ to $P_{j}$.

6. $P_{j}$ verifies the consistency of $c_{p}^{1}$.

If yes, he stores (sid, ssid, $P_{i}, P_{j}, C_{1}, \varepsilon, c_{p}^{2}$ ) and outputs (receipt, sid, ssid, $P_{i}, P_{j}$ ).

He ignores any later commitment messages with the same (sid, ssid) from $P_{i}$.

The decommit phase

Upon receiving a message (reveal, sid, ssid, $\left.P_{i}, P_{j}\right), P_{i}$ works as follows:

1. $P_{i}$ sends $\left(x, C_{2}, k_{2}, z\right)$ to $P_{j}$.

2. $P_{j}$ computes $m=G(x)$, and outputs (reveal, sid, ssid, $\left.P_{i}, P_{j}, x\right)$ if and only if $c_{p}^{2}$ is consistent and:

$$
g_{1}^{z}=\alpha u_{1}{ }^{\varepsilon}, g_{2}{ }^{z}=\beta u_{2}{ }^{\varepsilon}, h^{z}=\gamma(e / m)^{\varepsilon},\left(c d^{\omega}\right)^{z}=\delta v^{\varepsilon}
$$

Fig. 7. Our New UC-Secure Commitment Protocol Adaptive with Erasures

\subsection{Security Proof}

We now provide a full proof, with a sequence of games, that the above protocol emulates the ideal functionality against adaptive corruptions with erasures. This sequence starts from the real game, where the adversary interacts with real players, and ends with the ideal game, where we have built a simulator that makes the interface between the ideal functionality and the adversary.

As already explained, we denote by $C_{3}=C_{2} C_{1}{ }^{\varepsilon}$, the tuple involved in the last check. It should be a partial encryption of $m$ under randomness $z=s+\varepsilon r: C_{3}=\operatorname{PCS}(m ; \omega, z)$ where $\omega$ is the hash value of the first three terms of $C_{1}$.

Game $\mathbf{G}_{0}$ : This is the real game, in which every flow from the honest players is generated correctly by the simulator which knows the input $x$ sent by the environment to the sender. There is no use of the ideal functionality for the moment.

Game $\mathbf{G}_{1}$ : In this game, we focus on the simulation of an honest receiver interacting with a corrupted sender. Executions with an honest sender are still simulated as before, using the input $x$. The simulator 
will generate the CRS in such a way it knows the Cramer-Shoup decryption key, but $\zeta$ is a discrete logarithm challenge.

Upon receiving the values $\left(c_{p}^{1}, c_{p}^{2}\right)$ from the adversary, the simulator simply chooses a challenge $\varepsilon$ at random and sends it to the adversary, as $P_{j}$ would do with $P_{i}$. After receiving the values $\left(C_{1}, k_{1}\right)$, the simulator checks the consistency of the Pedersen commitment $c_{p}^{1}$ and aborts in case of failure. It then uses the Cramer-Shoup decryption key to recover the value $m^{\prime}$ sent by the adversary, and computes $x^{\prime}=G^{-1}\left(m^{\prime}\right)$. In case of invalid ciphertext, one sets $x^{\prime}=\perp$ (an element not in the domain of $G$ ). It stores (sid, ssid, $\left.P_{i}, P_{j}, C_{1}, \varepsilon, c_{p}^{2}\right)$ and $\left(x^{\prime}\right.$, sid, $P_{i}, P_{j}$ ) (this will correspond later to the Commit query to the ideal functionality, in the ideal game). Upon receiving the values $\left(x, C_{2}, k_{2}, z\right)$, the simulator does as $P_{j}$ would do in checking the commitment $c_{p}^{2}$ and that $C_{3}=\operatorname{PCS}\left(m^{\varepsilon} ; \omega, z\right)$, but accepts $x^{\prime}$ as the opening for the commitment.

The only difference with the previous game is that $P_{i}$ will accept $x^{\prime}$, as decrypted from $C_{1}=\operatorname{CS}\left(m^{\prime}=\right.$ $\left.G\left(x^{\prime}\right) ; r\right)$, for the decommitment instead of the value $x$ output at the decommitment time, which leads to $m=G(x)$ that matches with $C_{3}=\operatorname{PCS}\left(m^{\varepsilon} ; \omega, z\right)$, but that is also contained in $c_{p}^{2}$ together with $C_{2}$. We will show that under the binding property of the Pedersen commitment, one necessarily has $x^{\prime}=x$, and thus there is no difference.

Let us assume that $x^{\prime} \neq x$ in at least one of such executions: for the first one, we rewind the adversary up to the step 4., and send a new random challenge $\varepsilon^{\prime}$. Then the adversary should send the same $C_{1}$, otherwise one extracts the discrete logarithm of $\zeta$ in basis $g$ or a collision for $\mathfrak{H}_{K}$, and the same pair $\left(m, C_{2}\right)$ in the decommit phase for the same reason, but possibly a different $z^{\prime}$. Then, the final checks guarantee that $C_{3}=\operatorname{PCS}\left(m^{\varepsilon} ; \omega, z\right)$ in the first execution and $C_{3}^{\prime}=\operatorname{PCS}\left(m^{\varepsilon^{\prime}} ; \omega, z^{\prime}\right)$ in the second execution. From the homomorphic property: $C_{2}$ encrypts $\left(m / m^{\prime}\right)^{\varepsilon}$ in the first execution, but $\left(\mathrm{m} / \mathrm{m}^{\prime}\right)^{\varepsilon^{\prime}}$ in the second execution, which are thus equal. Since $\varepsilon^{\prime} \neq \varepsilon$, this implies that $m^{\prime}=m$. For the same reason, one can note that if $C_{1}$ is not a valid ciphertext, $C_{3}$ cannot be valid either (for the fixed $\omega$ ). We stress that the rewind here is just for the proof of indistinguishability of the two games, but not in the simulation.

In case of corruption of the receiver, one can note that he has no secret.

Game $\mathbf{G}_{2}$ : In this game, we start modifying the simulation of an honest sender, still knowing his input $x$. For the honest verifier against a corrupted sender, we still have to know the Cramer-Shoup decryption key to run the same simulation as in the previous game. But we now need to know the discrete logarithm for equivocating the Pedersen commitment.

This game is almost the same as the previous one excepted the way the double Cramer-Shoup ciphertext is generated: $\left(C_{1}, C_{2}\right)=\mathrm{DCS}(m, n ; r, s)$, for a random $n$ instead of 1 . The rest of the commit phase is unchanged.

At the decommit phase, $\mathcal{S}$ chooses random coins $z$ and computes $C_{3}=\operatorname{PCS}(m ; \omega, z)$, and then "repairs" $C_{2}=C_{3} / C_{1}{ }^{\varepsilon}$, and $k_{2}$ for being able to open $c_{p}^{2}$ to this new value.

Thanks to the homomorphic property, the repaired $C_{2}$ is indeed a correct ciphertext of 1 , and the equivocation of the Pedersen commitment guarantees a correct opening. This game is thus perfectly indistinguishable from the previous one.

In case a corruption of $P_{i}$ occurs before the decommit phase, the simulator anticipates the equivocation of $c_{p}^{2}$.

Game $\mathbf{G}_{3}$ : One can note that in the previous game, $r$ is not used anymore to compute $z$. One could thus ignore it, unless $\mathcal{P}_{i}$ gets corrupted before $\varepsilon$ has been sent, since we should be able to give it. But in such a case, one can compute again $C_{1}$ knowing $r$ and equivocate $c_{p}^{1}$.

We thus alter again the way the double Cramer-Shoup ciphertext is generated: $\left(C_{1}, C_{2}\right)=\operatorname{DCS}\left(m^{\prime}, n ; r, s\right)$, for random $m^{\prime}$ and $n$. Everything remains unchanged.

The unique change is thus the ciphertext $C_{1}$ that encrypts a random $m^{\prime}$ instead of $m$. One can run the IND-CCA security game, in an hybrid way, to show this game is indistinguishable from the previous one. To this aim, one has to show that the random coins $r$ are not needed to be known, and that the challenge ciphertexts are never asked for decryption (where the decryption key here is replaced by an access to the 
decryption oracle, hence the IND-CCA security game). The former point has been discussed above. For the latter, we have shown that the value actually encrypted in $C_{1}$ by the corrupted sender is the value sent at the decommit phase, which would even break the one-wayness of the encryption. Hence, if such a replay happens, one knows that the decommit phase will fail.

In case of corruption of $P_{i}$ before receiving $\varepsilon$, Pedersen commitments only have been sent, and they can thus be equivocated with correct values. In case of corruption of $P_{i}$ after having received $\varepsilon$, one does has before, anticipating the equivocation of $c_{p}^{2}$.

Game $\mathbf{G}_{4}$ : This is the ideal game, in which the simulator works as described below: when $P_{i}$ is corrupted, one uses the decryption of $C_{1}$ to send the Commit query to the ideal functionality, when $P_{i}$ is honest one can wait for the reveal information to conclude the simulation of the real flows.

\subsection{Description of the Simulator}

Setup. The simulator generates the parameters, knowing the Cramer-Shoup decryption key and the Pedersen equivocation trapdoor.

\section{When $P_{i}$ is honest.}

COMmit STAGE: Upon receiving the information that a commitment has been performed, with (receipt, sid, ssid, $\left.P_{i}, P_{j}\right)$ from $\mathcal{F}_{\text {mcom }}, \mathcal{S}$ computes $\left(C_{1}, C_{2}\right)=\operatorname{DCS}\left(m^{\prime}, n ; r, s\right)$, for random $m^{\prime}$ and $n$ but then follows as $P_{i}$ would do. If $P_{j}$ is honest too, one just has to send a random $\varepsilon$.

In case of corruption of $P_{i}$ before receiving $\varepsilon$, one can equivocate $c_{p}^{1}$, otherwise one equivocates $c_{p}^{2}$, as explained below.

DECOMMIT STAGE: Upon receiving the information that the decommitment has been performed on $x$, with (reveal, sid, ssid, $P_{i}, P_{j}, x$ ) from $\mathcal{F}_{\text {mcom }}, \mathcal{S}$ exploits the equivocability of the Pedersen commitment: it first chooses a random $z$ and computes the ciphertext $C_{3}=\operatorname{PCS}(m=G(x) ; \omega, z)$. It then adapts $C_{2}=C_{3} / C_{1} \varepsilon$ and uses the trapdoor for the Pedersen commitment to produce a new value $k_{2}$ corresponding to the new value $C_{2}$. It then simulates the decommit phase to $P_{j}$.

When $P_{i}$ is corrupted and $P_{j}$ is honest.

Commit stage: Upon receiving $\left(C_{1}, k_{1}\right)$ from the adversary, $\mathcal{S}$ decrypts the Cramer-Shoup ciphertext $C_{1}$ and extracts $x$ from $G$. If the decryption is invalid, then $\mathcal{S}$ sends (Commit, sid, ssid, $P_{i}, P_{j}, \perp$ ) to $\mathcal{F}_{\text {mcom }}$. Otherwise, $\mathcal{S}$ sends (Commit, sid, ssid, $P_{i}, P_{j}, x$ ).

Decommit Stage: $\mathcal{S}$ acts as a regular honest user $P_{j}$ from the incoming message of $\mathcal{A}$ on behalf of $P_{i}$. In case of validity, send the query (reveal, sid, ssid).

\section{Acknowledgment}

We thank Yehuda Lindell for his fruitful comments.

\section{References}

[ACP09] Michel Abdalla, Céline Chevalier, and David Pointcheval. Smooth projective hashing for conditionally extractable commitments. In Shai Halevi, editor, Advances in Cryptology - CRYPTO 2009, volume 5677 of Lecture Notes in Computer Science, pages 671-689. Springer, August 2009.

$\left[\mathrm{BBC}^{+} 13\right]$ Fabrice Ben Hamouda, Olivier Blazy, Céline Chevalier, David Pointcheval, and Damien Vergnaud. Efficient ucsecure authenticated key-exchange for algebraic languages. In Kaoru Kurosawa, editor, Proceedings of PKC 2013 , Lecture Notes in Computer Science. Springer, 2013. Full version available from the web page of the authors or from http://eprint.iacr.org/2012/284.

$\left[\mathrm{BFI}^{+} 10\right]$ Olivier Blazy, Georg Fuchsbauer, Malika Izabachène, Amandine Jambert, Hervé Sibert, and Damien Vergnaud. Batch Groth-Sahai. In Jianying Zhou and Moti Yung, editors, ACNS 10: 8th International Conference on Applied Cryptography and Network Security, volume 6123 of Lecture Notes in Computer Science, pages 218-235. Springer, June 2010. 
The commmit phase

$$
\begin{gathered}
m=G\left(x, \text { sid, ssid, } P_{i}, P_{j}\right) \\
r \stackrel{\$}{\leftarrow} \mathbb{Z}_{p}, k_{1} \stackrel{\$}{\leftarrow} \mathbb{Z}_{p} \\
C=\operatorname{CS}(m ; r) \\
c_{p}^{1}=\operatorname{Ped}\left(\mathfrak{H}_{K}(C) ; k_{1}\right) \\
s \stackrel{\$}{\leftarrow} \mathbb{Z}_{p}, k_{2} \stackrel{\$}{\leftarrow} \mathbb{Z}_{p} \longleftarrow, S \stackrel{\$}{\leftarrow} \mathbb{Z}_{p}, \varepsilon \stackrel{\$}{\leftarrow}\{0,1\}^{n} \\
(\alpha, \beta, \gamma, \delta)=\left(g_{1}{ }^{s}, g_{2}{ }^{s}, h^{s},\left(c d^{\omega}\right)^{s}\right) \\
c_{p}^{2}=\left(g_{1}{ }^{R} g_{2}{ }^{S}, h_{1}{ }^{R} h_{2}{ }^{S} G(\varepsilon)\right) \\
\left.\operatorname{Ped}\left(\mathfrak{H}_{K}(\alpha, \beta, \gamma, \delta)\right) ; k_{2}\right)
\end{gathered}
$$

The decommmit phase

$$
\begin{aligned}
\stackrel{\left(x, \alpha, \beta, \gamma, \delta, k_{2}, z\right)}{\longrightarrow} & m=G\left(x, \text { sid, ssid, } P_{i}, P_{j}\right) \\
& \text { checks } c_{p}^{2} \text { and whether } \\
& g_{1}^{z}=\alpha u_{1}{ }^{\varepsilon}, g_{2}{ }^{z}=\beta u_{2}{ }^{\varepsilon} \\
& h^{z}=\gamma(e / m)^{\varepsilon},\left(c d^{\omega}\right)^{z}=\delta v^{\varepsilon}
\end{aligned}
$$

The commmit phase

$$
\begin{gathered}
m=G(x) \\
r \stackrel{\$}{\leftarrow} \mathbb{Z}_{p}, s \stackrel{\$}{\leftarrow} \mathbb{Z}_{p} \\
k_{1}, k_{2} \stackrel{\$}{\leftarrow} \mathbb{Z}_{p} \\
\left(C_{1}, C_{2}\right)=\operatorname{DCS}(m ; 1 ; r, s) \\
c_{p}^{1}=\operatorname{Ped}\left(\mathfrak{H}_{K}\left(C_{1}\right) ; k_{1}\right) \\
c_{p}^{2}=\operatorname{Ped}\left(\mathfrak{H}_{K}\left(C_{2}, m, \operatorname{sid}, \operatorname{ssid}, P_{i}, P_{j}\right) ; k_{2}\right) \frac{c_{p}^{1}, c_{p}^{2}}{\varepsilon} \\
z=s+\varepsilon r, \text { erases } r, s \stackrel{k_{1}, C_{1}}{\leftarrow} \mathbb{Z}_{p} \\
\text { Aborts if } c_{p}^{1} \\
\text { inconsistent }
\end{gathered}
$$

The decommmit phase

$$
\begin{aligned}
\longrightarrow & m=G(x) \\
& \text { checks } c_{p}^{2} \text { and whether } \\
& g_{1}^{z}=\alpha u_{1}{ }^{\varepsilon}, g_{2}{ }^{z}=\beta u_{2}{ }^{\varepsilon} \\
& h^{z}=\gamma(e / m)^{\varepsilon},\left(c d^{\omega}\right)^{z}=\delta v^{\varepsilon}
\end{aligned}
$$

Fig. 8. Comparison of Lindell's Adaptive Protocol (above) and Ours (below)

[Can01] Ran Canetti. Universally composable security: A new paradigm for cryptographic protocols. In 42nd Annual Symposium on Foundations of Computer Science, pages 136-145. IEEE Computer Society Press, October 2001.

[CF01] Ran Canetti and Marc Fischlin. Universally composable commitments. In Joe Kilian, editor, Advances in Cryptology - CRYPTO 2001, volume 2139 of Lecture Notes in Computer Science, pages 19-40. Springer, August 2001.

$\left[\mathrm{CHK}^{+} 05\right]$ Ran Canetti, Shai Halevi, Jonathan Katz, Yehuda Lindell, and Philip D. MacKenzie. Universally composable password-based key exchange. In Ronald Cramer, editor, Advances in Cryptology - EUROCRYPT 2005, volume 3494 of Lecture Notes in Computer Science, pages 404-421. Springer, May 2005.

[CLOS02] Ran Canetti, Yehuda Lindell, Rafail Ostrovsky, and Amit Sahai. Universally composable two-party and multiparty secure computation. In 34th Annual ACM Symposium on Theory of Computing, pages 494-503. ACM Press, May 2002.

[CS98] Ronald Cramer and Victor Shoup. A practical public key cryptosystem provably secure against adaptive chosen ciphertext attack. In Hugo Krawczyk, editor, Advances in Cryptology - CRYPTO'98, volume 1462 of Lecture Notes in Computer Science, pages 13-25. Springer, August 1998.

[CS03] Jan Camenisch and Victor Shoup. Practical verifiable encryption and decryption of discrete logarithms. In Dan Boneh, editor, Advances in Cryptology - CRYPTO 2003, volume 2729 of Lecture Notes in Computer Science, pages 126-144. Springer, August 2003. 
[DN02] Ivan Damgård and Jesper Buus Nielsen. Perfect hiding and perfect binding universally composable commitment schemes with constant expansion factor. In Moti Yung, editor, Advances in Cryptology - CRYPTO 2002, volume 2442 of Lecture Notes in Computer Science, pages 581-596. Springer, August 2002.

[FLM11] Marc Fischlin, Benoît Libert, and Mark Manulis. Non-interactive and re-usable universally composable string commitments with adaptive security. In Advances in Cryptology - ASIACRYPT 2011, Lecture Notes in Computer Science, pages 468-485. Springer, December 2011.

[GL03] Rosario Gennaro and Yehuda Lindell. A framework for password-based authenticated key exchange. In Eli Biham, editor, Advances in Cryptology - EUROCRYPT 2003, volume 2656 of Lecture Notes in Computer Science, pages 524-543. Springer, May 2003. http://eprint.iacr.org/2003/032.ps.gz.

[GS08] Jens Groth and Amit Sahai. Efficient non-interactive proof systems for bilinear groups. In Nigel P. Smart, editor, Advances in Cryptology - EUROCRYPT 2008, volume 4965 of Lecture Notes in Computer Science, pages $415-432$. Springer, April 2008.

[Lin11a] Yehuda Lindell. Highly-efficient universally-composable commitments based on the DDH assumption. In Kenneth G. Paterson, editor, Advances in Cryptology - EUROCRYPT 2011, volume 6632 of Lecture Notes in Computer Science, pages 446-466. Springer, May 2011.

[Lin11b] Yehuda Lindell. Highly-efficient universally-composable commitments based on the ddh assumption. Cryptology ePrint Archive, Report 2011/180, 2011. http://eprint.iacr.org/.

[NY89] Moni Naor and Moti Yung. Universal one-way hash functions and their cryptographic applications. In 21st Annual ACM Symposium on Theory of Computing, pages 33-43. ACM Press, May 1989.

[Ped92] Torben P. Pedersen. Non-interactive and information-theoretic secure verifiable secret sharing. In Joan Feigenbaum, editor, Advances in Cryptology - CRYPTO'91, volume 576 of Lecture Notes in Computer Science, pages 129-140. Springer, August 1992.

[Yao82] Andrew C. Yao. Theory and applications of trapdoor functions. In 23rd Annual Symposium on Foundations of Computer Science, pages 80-91. IEEE Computer Society Press, November 1982. 